\title{
Erratum to: Stability of triangular equilibrium points in the elliptic restricted three body problem with oblate and triaxial primaries
}

\section{F.A. Abd El-Salam ${ }^{1,2}$}

Published online: 13 May 2015

(C) Springer Science+Business Media Dordrecht 2015

Erratum to: Astrophys Space Sci (2015) 357:15

DOI 10.1007/s10509-015-2308-5

There is an error in Eq. (1) in the original publication. The symbol $f$ should have been in the denominators of both second terms in the first part of that equation. The whole correct equation is shown below.

$\frac{d^{2} \xi}{d f^{2}}-2 \frac{d \eta}{d f}=(1+e \cos f)^{-1} \frac{\partial U}{\partial \xi}$,

$\frac{d^{2} \eta}{d f^{2}}+2 \frac{d \xi}{d f}=(1+e \cos f)^{-1} \frac{\partial U}{\partial \eta}$

where

$$
\begin{aligned}
U= & \frac{1}{2}\left[(1-\mu) r_{1}^{2}+\mu r_{2}^{2}\right]+\frac{1}{n^{2}}\left[\frac{(1-\mu)}{r_{1}}+\frac{A_{1}(1-\mu)}{2 r_{1}^{3}}\right. \\
& \left.+\frac{(1-\mu)\left(2 \sigma_{1}-\sigma_{2}\right)}{2 r_{1}^{3}}+\frac{\mu}{r_{2}}+\frac{A_{2} \mu}{2 r_{2}^{3}}+\frac{\mu\left(2 \gamma_{1}-\gamma_{2}\right)}{2 r_{2}^{3}}\right]
\end{aligned}
$$

The online version of the original article can be found under doi:10.1007/s10509-015-2308-5.

$\triangle$ F.A. Abd El-Salam

f.a.abdelsalam@gmail.com

1 Department of Mathematics, Faculty of Science, Taibah University, Madina, Saudi Arabia

2 Department of Astronomy, Faculty of Science, Cairo University, Cairo, 12613, Egypt 